\title{
Synthesis of Water-Soluble Iridium (III)-Containing Nanoparticles for Biological Applications
}

\author{
Huanzhi Hou, ${ }^{1}$ Pengfei Sun, ${ }^{1}$ Quli Fan, ${ }^{1}$ Xiaomei Lu, ${ }^{1,2}$ Cheng Xue, ${ }^{1}$ Yixue Zhang, \\ Sichao Tian, ${ }^{1}$ and Wei Huang ${ }^{1,2}$ \\ ${ }^{1}$ Key Laboratory for Organic Electronics \& Information Displays (KLOEID) and Institute of Advanced Materials (IAM), \\ Nanjing University of Posts \& Telecommunications (NUPT), Nanjing 210046, China \\ ${ }^{2}$ Jiangsu-Singapore Joint Research Center on Organic/Bio-Electronics \& Information Displays and Institute of Advanced Materials, \\ Nanjing Tech University, Nanjing 211816, China
}

Correspondence should be addressed to Pengfei Sun; iampfsun@njupt.edu.cn and Wei Huang; iamdirector@fudan.edu.cn

Received 27 August 2015; Accepted 20 October 2015

Academic Editor: Jin Geng

Copyright (C) 2015 Huanzhi Hou et al. This is an open access article distributed under the Creative Commons Attribution License, which permits unrestricted use, distribution, and reproduction in any medium, provided the original work is properly cited.

\begin{abstract}
Water-soluble nanoparticles (Ir/PGlc-NP, Ir/ $\beta$-1,3-glucan-NP) based on water-soluble glycopolymers (PGlc), $\beta$-1,3-glucan polysaccharide, and conjugated phosphorescent Ir (III) complexes were successfully synthesized by self-assembly. The obtained nanoparticles have good spherical morphological characterization with a mean diameter of $50 \mathrm{~nm}$ measured by TEM. Ir/PGlc-NP and $\mathrm{Ir} / \beta$-1,3-glucan-NP showed the same emission maxima at $565 \mathrm{~nm}$ in aqueous solution and both caused effective apoptosis and death of HepG2 and Hela cells after being irradiated at $445 \mathrm{~nm}$ for $30 \mathrm{~min}$ in vitro. Fluorescence cellular imaging was conducted by confocal laser scanning microscopy (CLSM) using HepG2 cells as the model cell in which the nanoparticles had successfully entered into the cytoplasm with high brightness. Furthermore, after injecting the nanoparticles into live mice in vivo, the realtime fluorescence imaging as well as the nanoparticles distribution in organs at 24 hours after administration indicated that these nanoparticles can serve as fluorescent imaging contrast for further biological applications.
\end{abstract}

\section{Introduction}

In recent years, water-soluble fluorescent $\pi$-conjugated nanoparticles (WCPNs) have received much attention in bioimaging [1-4], drug-delivery, and sensing applications due to their high fluorescent brightness, excellent biocompatibility, and photostability [5-7]. The preparation of WCPNs was mainly focused on self-assembly method, because this method could retain the physical properties of the polymers. Our group has got some achievements in this field. For example, Lu et al. developed kinds of water-soluble nanoparticles using oligos ( $p$-phenyleneethynylene) (OHOPEL) and three water-soluble polymers (PEG, PAA, and PG) by noncovalent bond self-assembly [8]. Zhang et al. synthesized types of water-soluble fluorescent nanoparticles, and the constituent conjugated polymer chains of them showed without distinct aggregation [9]. Other groups like Moon et al. [10-12] demonstrated that the aggregation structure of the conjugated polymer nanoparticles can be changed by different organic acids treatment and that the water-soluble nanoparticles can be fabricated via controlled aggregation of semiflexible poly(phenylenebutadiynylene) with linear polysaccharide.

Phosphorescent Ir (III) complexes have attracted considerable interest during the past decade because of their excellent properties when used as energy receptor in organic light-emitting devices, chemical detection, and protein detection. Shi et al. [13] designed and synthesized phosphorescent conjugated polyelectrolyte with different contents of Ir (III) complex and demonstrated the applications of conjugated polyelectrolytes (CPEs) in time-resolved luminescent biodetection and fluorescence lifetime imaging. Compared with various organic fluorescent dyes units, Ir (III) complexes show unique advantages, for example, long fluorescent lifetime, large Stokes shift, high phosphorescent quantum yield, excellent photostability, tunable wavelengths, and easy modification of ligand structures [14-16]. These characteristics make Ir (III) complexes the most successful candidates in optical sensing and bioimaging applications 


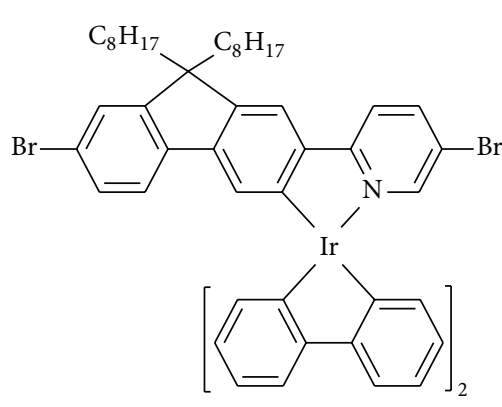

$\operatorname{Ir}(\text { ppy })_{3}$<smiles>CCOC(=O)C(C)(C)CC(C)(C)C(=O)OCC(O)Cn1cc(COC)nn1</smiles>

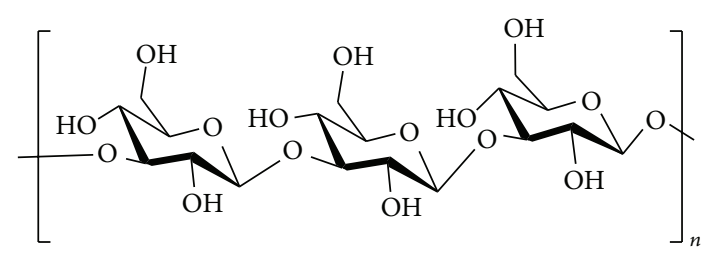

$\beta$-1,3-Glucan<smiles>OCC1OC(O)C(O)C(O)C(O)C1O</smiles>

(PGlc)

Scheme 1: Iridium (III) complexes Ir (ppy) ${ }_{3}$ and water-soluble glycopolymer, polysaccharide for the preparation of water-soluble fluorescent $\pi$-conjugated nanoparticles.

in complicated media. Our group has systematically studied conjugated polymers containing Ir (III) complexes for years in the application of biosensing, optical oxygen sensing, and photodynamic therapy [15-19]. Therefore, it is reasonable to introduce different amounts of the Ir (III) complex into the conjugated polymers backbone as the energy acceptor to acquire different phosphorescent nanoparticles.

Polysaccharide refers to any of a class of carbohydrates whose molecules contain chains of monosaccharide molecules. In recent years, Twomey et al. [11] utilized hyaluronic acid (HA) as hydrophilic group to obtain coreshell nanoparticles, and HA has specific binding ability with cell surface receptors. Glycopolymer is a synthetic polymer with pendant carbohydrate and various reports have concerned glycopolymer-based nanoparticles due to the amphiphilic properties of glycopolymer [20-23]. In this work, we reported that different water-soluble phosphorescent nanoparticles containing the Ir (III) complexes were obtained when $\pi$-conjugated polymers were treated with glycopolymer (poly(glucose)) or polysaccharide $(\beta-1,3$ glucan) based on self-assembly. Because of the amphiphilic structures, the polymers formed nanoparticles with the size around $50 \mathrm{~nm}$ in aqueous solution without additional decoration. Moreover, we studied the nanoparticles from the point of morphological characterization, optical properties, and cellular imaging. Finally, we analyzed the distribution of Ir/PGlc-NP and Ir/ $\beta$-1,3-glucan-NP in different organs of the mice at $24 \mathrm{~h}$ after administration. This study provided a possibility for further investigation on the application of photodynamic therapy.

\section{Experimental}

2.1. Materials. The iridium (III) complexes (Ir (ppy) $)_{3}$, poly(glucose) (PGlc), and polysaccharide ( $\beta$-1,3-glucan) were readily synthesized according to the literature [16, 24]. The structures of them were shown in Scheme 1.

2.2. Preparation of Core-Shell Nanoparticles. The strategy for fabricating WCPNs was based on self-assembly method; typically, iridium complex (Ir (ppy) ${ }_{3}$ ) and water-soluble glycopolymer (PGlc) were solubilized in DMF $(0.1 \mathrm{mg} / \mathrm{mL})$, respectively, and then $5 \mathrm{~mL}$ DMF solution was quickly injected into $5 \mathrm{~mL}$ water under ultrasonication. After being dialyzed against water for 2 days, getting the pale yellow clear aqueous solutions, the nanoparticle was denoted as Ir/PGlcNP. Similarly, Ir/ $\beta$-1,3-glucan-NP nanoparticles formed via the self-assembly of Ir (ppy) ${ }_{3}$ and polysaccharide (glucan).

2.3. Instrumentation. Hydrodynamic radius of the core-shell nanoparticles was measured by dynamic light scattering (DLS) using particle size analyzer (ALV/5000E) at a fixed scattering angle of $90^{\circ}$. UV-visible spectra were recorded with a Shimadzu UV-3150 UV-VIS-NIR spectrophotometer. Transmission electron microscopy (TEM) experiments were conducted using a Philips CM 120 electron microscope operated with accelerating voltage of $80 \mathrm{kV}$. A small drop of a water-soluble nanoparticles solution was added to a carbon-coated copper grid. Drying for several hours, TEM measurements can be performed. Photoluminescent spectra (PL) were obtained on an FLS 920 spectrofluorophotometer which was purchased from Edinburgh Instruments. Confocal laser scanning microscope (CLSM) images were taken using a Leica TCS SP5 microscope.

\section{Results and Discussion}

3.1. Morphological Characterization. First, dynamic light scattering (DLS) was used to characterize the sample solutions, and the diameter distribution was shown in Figure 1. It 


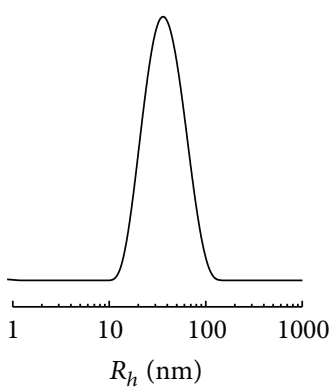

(a)

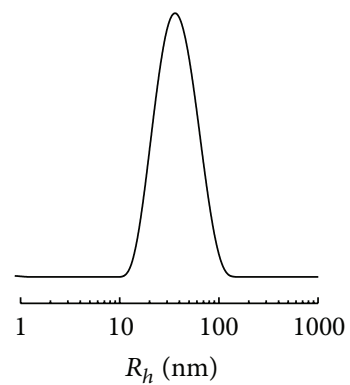

(b)

FIGURE 1: Hydrodynamic radius distribution graph of nanoparticles: (a) Ir/PGlc-NP; (b) Ir/ $\beta$-1,3-glucan-NP.

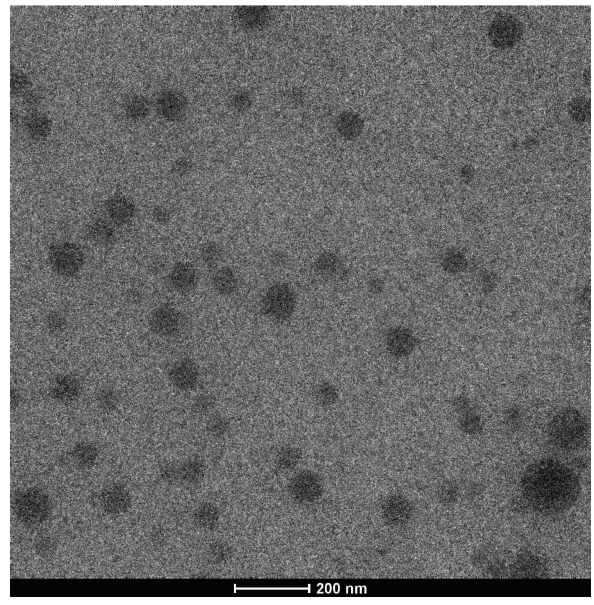

(a)

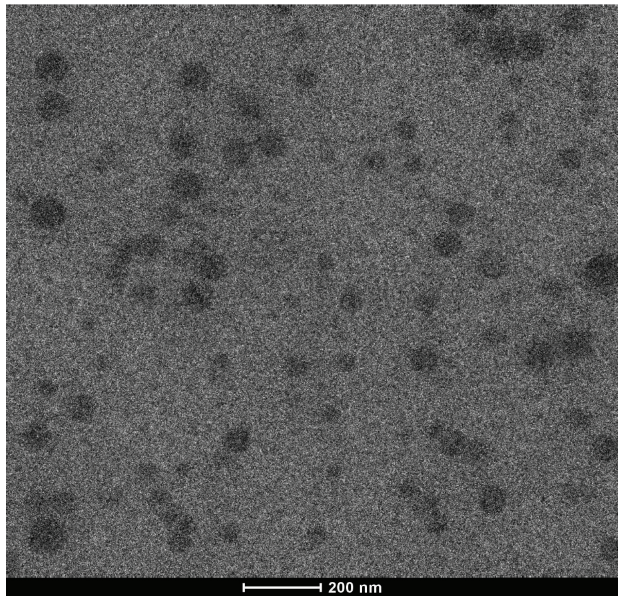

(b)

FIGURE 2: Representative TEM images of particles (a) Ir/PGlc-NP and (b) Ir/ $\beta$-1,3-glucan-NP prepared by freeze drying.

TABLE 1: DLS characterization data of the nanoparticles prepared by $\operatorname{Ir}(\text { ppy })_{3}$ and PGlc or $\beta$-1,3-glucan.

\begin{tabular}{lcc}
\hline Sample & $\left\langle R_{h}\right\rangle(\mathrm{nm})$ & Polydispersity index (PI) \\
\hline Ir/PGlc-NP & 33 & 0.14 \\
Ir/ $\beta$-1,3-glucan-NP & 31 & 0.22 \\
\hline
\end{tabular}

was clear that the average hydrodynamic radius $\left(R_{h}\right)$ mostly ranged from 10 to $100 \mathrm{~nm}$. Table 1 gave the detailed data about $R_{h}$ and PI, which revealed that the average diameter was about $60 \mathrm{~nm}$ with narrow size distribution as PI of around 0.2 .

TEM Analysis. The size and morphology of the Ir/PGlcNP and Ir/ $\beta$-1,3-glucan-NP in solid state were studied using TEM. The pictures, obtained by freeze drying, were shown in Figure 2. The pictures showed the two sample nanoparticles have similar structure.

In Figure 2, both the Ir/PGlc-NP and the Ir/ $\beta$-1,3-glucanNP samples showed good spherical morphology with average diameters of $\sim 50 \mathrm{~nm}$. Because the density of coordination complex Ir (ppy) ${ }_{3}$ and PGlc/ $\beta$-1,3-glucan was the same and consequently the same contrasted to the background, there was no evidence to distinguish them from the pictures.
However, the diameters observed were smaller than those obtained from DLS $\left(\left\langle R_{h}\right\rangle=33 \mathrm{~nm}\right)$ mostly because the nanoparticles were shrunk during the process of freeze drying. The DLS and TEM results show that the nanoparticles (Ir/PGlc-NP and Ir/ $\beta$-1,3-glucan-NP) have spherical morphology, with the water-soluble polymer (PGlc and $\beta$-1,3glucan) as the shell and $\operatorname{Ir}(\mathrm{ppy})_{3}$ as the core. Also, diameter of nanoparticle (Ir/PGlc-NP and Ir/ $\beta$-1,3-glucan-NP) was about $50 \mathrm{~nm}$, which was much smaller than our previous reports $[8,9]$.

3.2. Optical Properties. The photoluminescence (PL) emission spectra of $\operatorname{Ir}(\mathrm{ppy})_{3}$ in THF, Ir/PGlc-NP, and $\operatorname{Ir} / \beta$ 1,3-glucan-NP in aqueous solution are shown in Figure 3. The photoluminescence emission spectra of nanoparticles and $\operatorname{Ir}$ (ppy) $)_{3}$ in THF were similar without distinct blueshift or red-shift and their emission maximum was around $565 \mathrm{~nm}$. Compared with other nanoparticles with conjugated polymers as the core, which can induce PL changes for aggregation, our nanoparticles showed good optical stability. This proved that, after self-assembly of the particles formed in water, there was no significant change.

3.3. Cellular Imaging. In order to demonstrate the ability of Ir/PGlc-NP and Ir/ $\beta$-1,3-glucan-NP as a probe for 


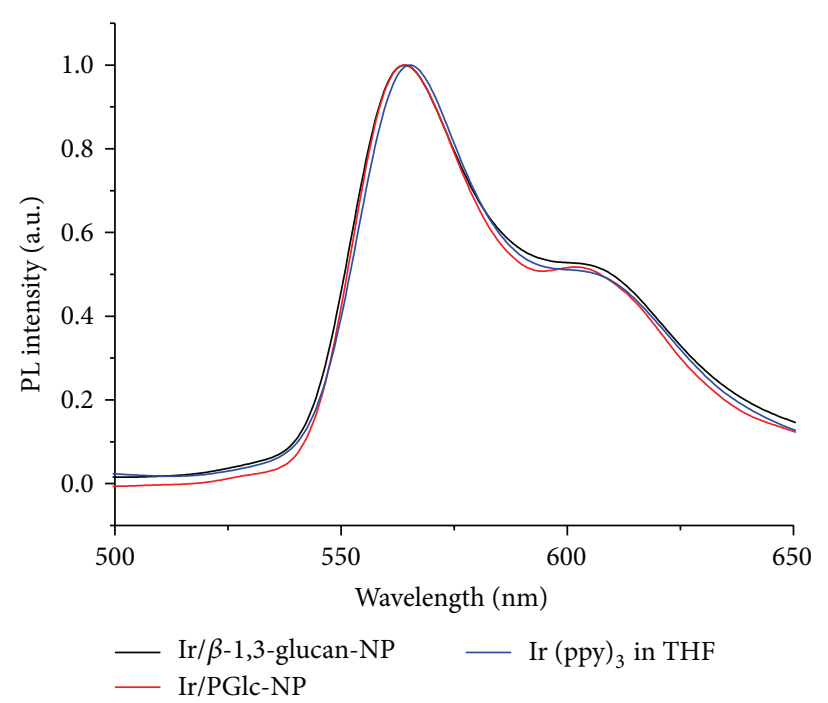

Figure 3: Normalized PL spectra of Ir (ppy) $)_{3}$ in THF, Ir/PGlc-NP, and Ir/ $\beta$-1,3-glucan-NP.

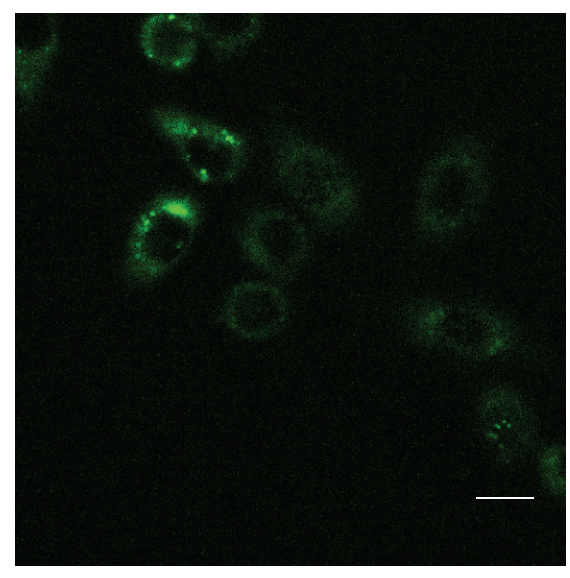

(a1)

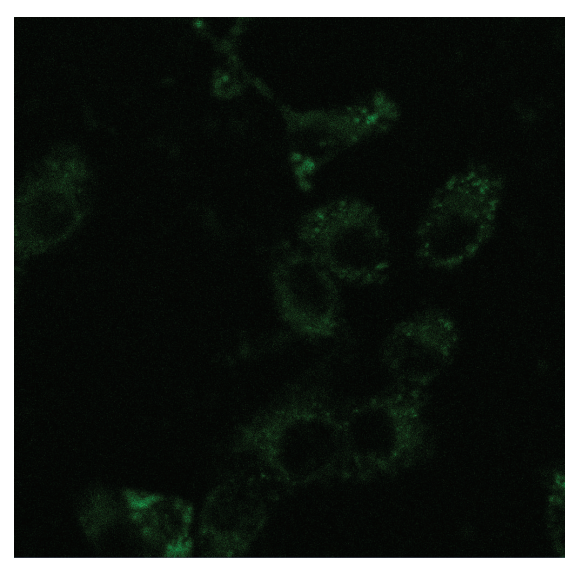

(a2)

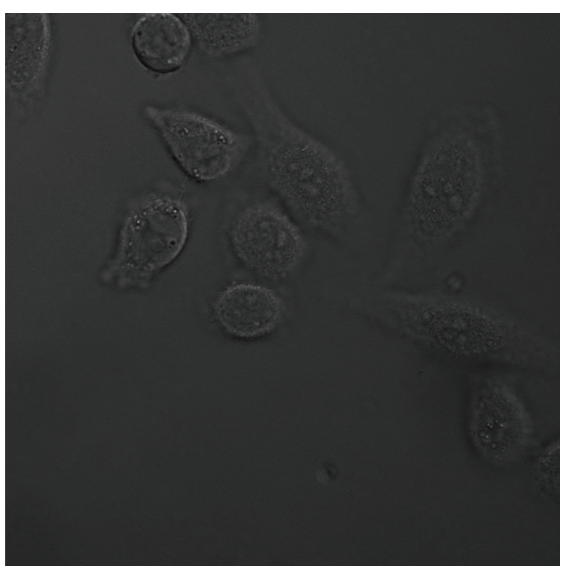

(b1)

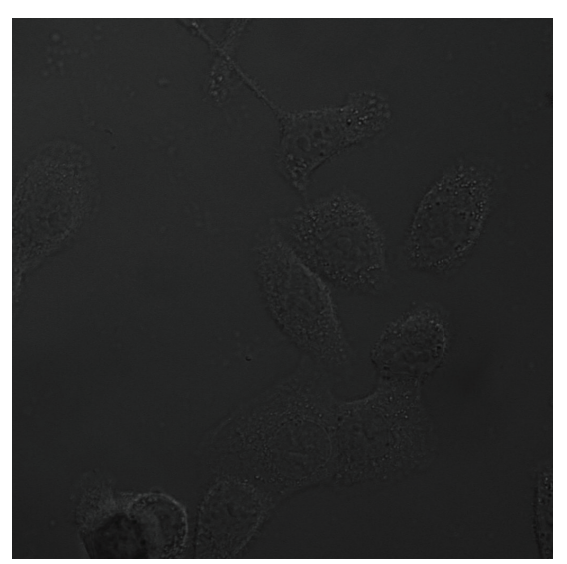

(b2)

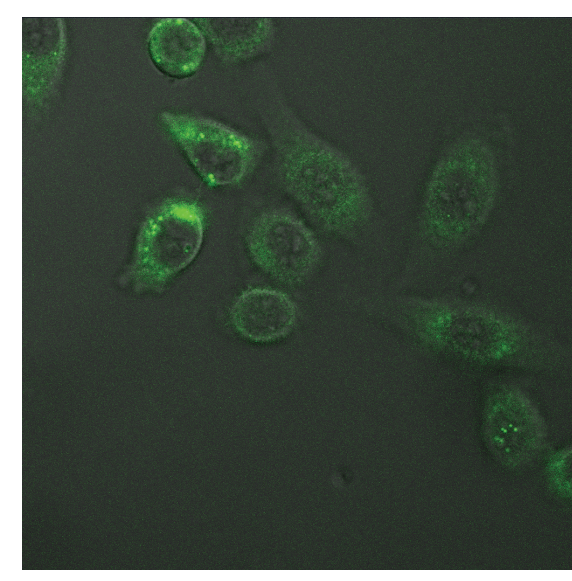

(c1)

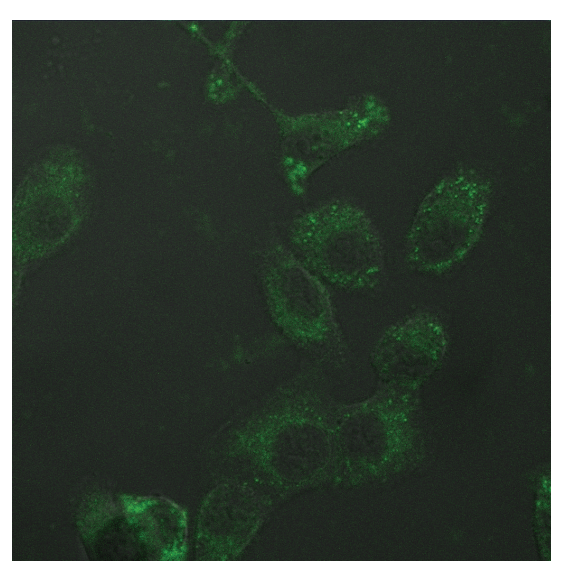

(c2)

FIGURE 4: CLSM images of HepG2 cells incubated with the nanoparticles Ir/PGlc-NP(1) and Ir/ $\beta$-1,3-glucan-NP(2) at $37^{\circ} \mathrm{C}$ for $2 \mathrm{~h}$. (a) Fluorescence images of the nanoparticles, (b) phase contrast bright-field image, and (c) overlay images of the nanoparticles and the blank HepG2 cells. Scale bar of all the images was the same: $10 \mu \mathrm{m}$. 


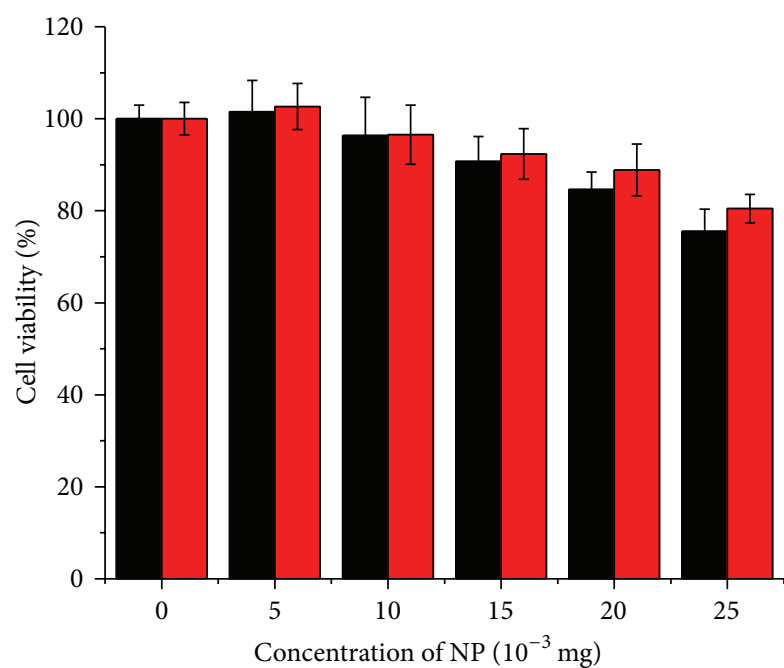

Concentration of NP $\left(10^{-3} \mathrm{mg}\right)$

Ir/PGlc-NP

$\operatorname{Ir} / \beta$-1,3-glucan

(a)

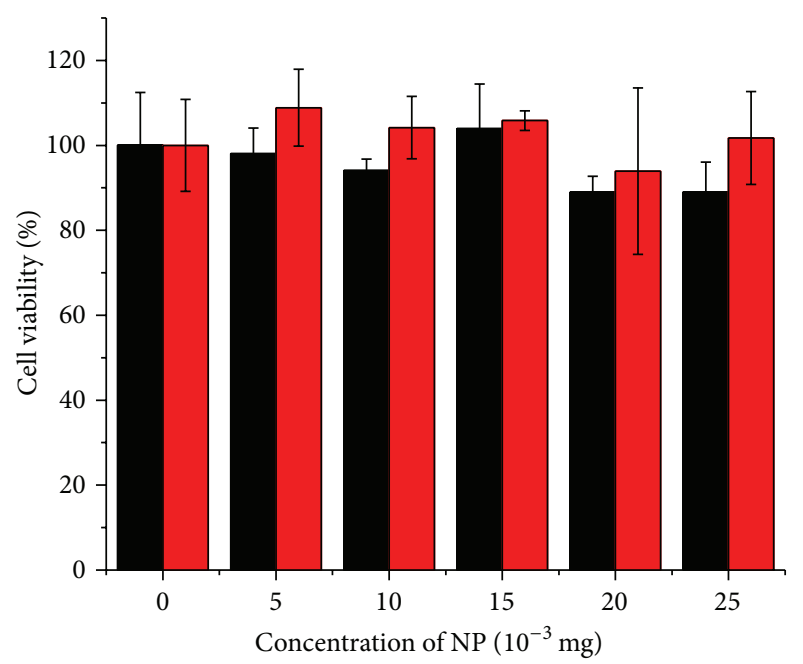

Ir/PGlc-NP

$\operatorname{Ir} / \beta$-1,3-glucan

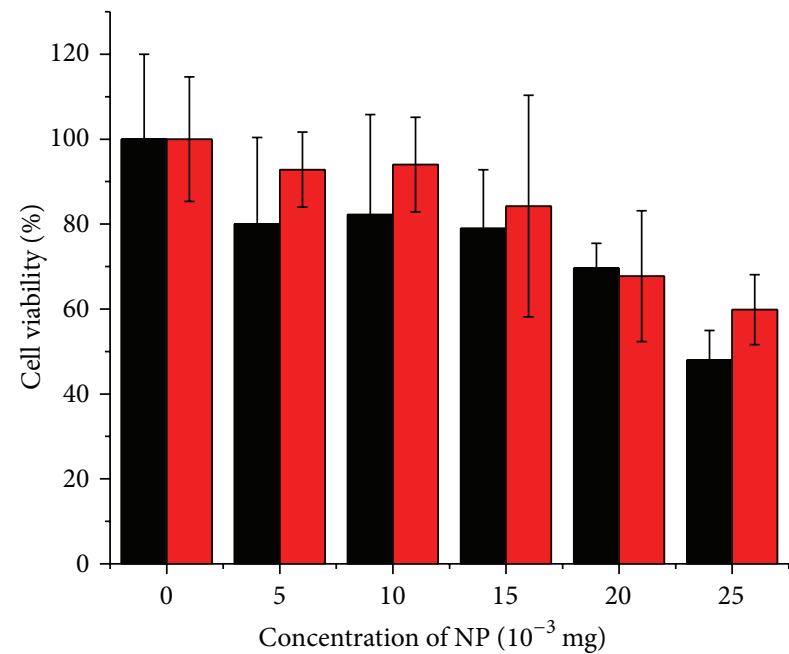

Ir/PGlc-NP

Ir/ $\beta$-1,3-glucan

(b)

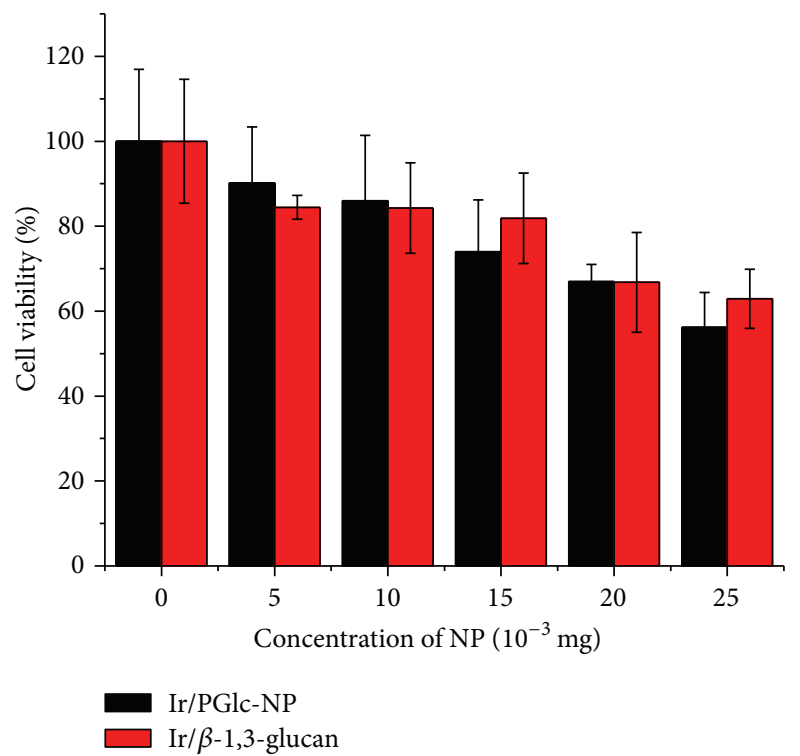

(d)

FIGURE 5: In vitro photodynamic cytotoxicity experiment of Ir/PGlc-NP and Ir/ $\beta$-1,3-glucan-NP against HepG2 (a, b) and Hela (c, d) cells incubated in the dark (a, c) and irradiated at $445 \mathrm{~nm}$ for $30 \mathrm{~min}(\mathrm{~b}, \mathrm{~d})$, respectively.

cellular imaging in vitro, human hepatocellular carcinoma cells (HepG2) were selected as the model with the purpose of fluorescence imaging by confocal laser scanning microscopy (CLSM). After being coincubated with Ir/PGlc$\mathrm{NP}$ and $\mathrm{Ir} / \beta-1,3$-glucan-NP at $37^{\circ} \mathrm{C}$ for $2 \mathrm{~h}$, HepG 2 cells were fixed. Figure 4(a) showed the fluorescence images of HepG2 cells stained with Ir/PGlc-NP and Ir/ $\beta$-1,3-glucan-NP, respectively. Figure 4 (b) showed the phase contrast brightfield image and Figure 4(c) exhibited overlay images of the nanoparticles and the HepG2 cells. Strong fluorescence signals were observed throughout the whole cytoplasm of the HepG2 cells in Figures 4(a) and 4(c). However, the nucleus has little fluorescence, implying that Ir/PGlc-NP and $\mathrm{Ir} / \beta$-1,3-glucan-NP can be efficiently internalized through endocytosis mechanism by HepG2 cells and that the fluorescence was derived from Ir/PGlc-NP and Ir/ $\beta$-1,3-glucanNP rather than autofluorescence from the blank HepG2 cells. These results further demonstrated the nanoparticles can be used in cell imaging.

3.4. Photocytotoxicity Test. In order to prove the application potential of Ir/PGlc-NP and Ir/ $\beta$-1,3-glucan-NP in photodynamic therapy (PDT), next the photodynamic cytotoxicity experiment of these two nanoparticles against HepG2 and Hela cells was evaluated by traditional MTT assay [15]. The nanoparticles with a series of concentrations $(2.5,5,10,20$, and $50 \mu \mathrm{g}$ ) were tested in the dark and under light irradiation at $445 \mathrm{~nm}$ for $30 \mathrm{~min}$. As shown in Figures 5(a) and 5(c), 


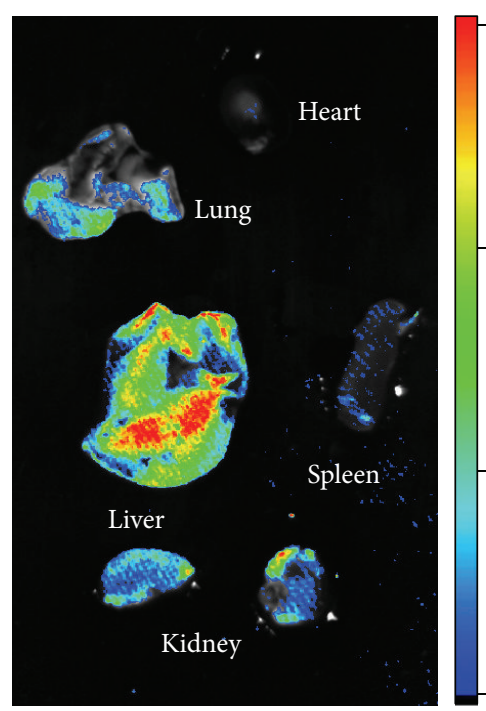

(a)

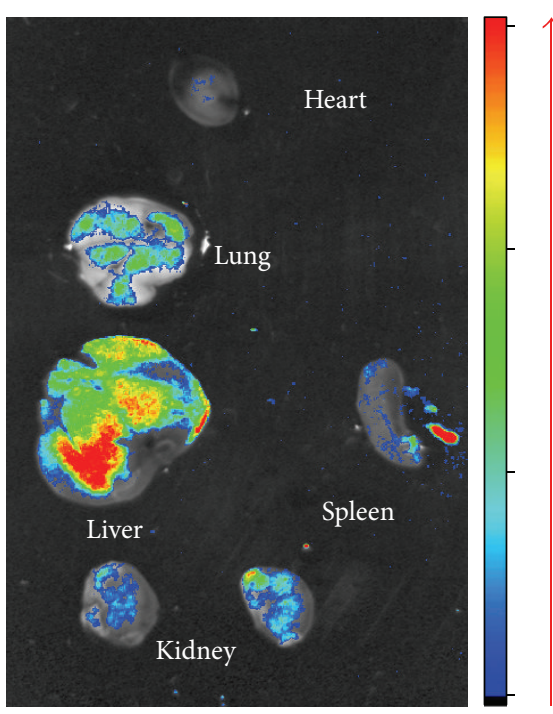

(b)

FIGURE 6: Fluorescence images of the organs of the mice after intravenous injection of nanoparticles via the tail: (a) Ir/PGlc-NP; (b) Ir/ $\beta$-1,3glucan-NP at $24 \mathrm{~h}$ after administration.

nanoparticles showed slight toxicity over an incubation period for $24 \mathrm{~h}$ with HepG2/Hela cells in the dark because the PGlc/-1,3-glucan as the shell of the nanoparticles reduced the toxicity of Ir (III) complexes. As shown in Figures 5(b) and 5(d), cell viability of HepG2 and Hela cells rapidly decreased along with the increase of the nanoparticles concentration and about $50 \%$ of the cell killing was observed when the concentration of nanoparticles was up to $50 \mu \mathrm{g}$ under irradiation for $30 \mathrm{~min}$. It was obviously observed from the images that the anticancer ability of the two nanoparticles was similar. These results manifested that Ir/PGlc-NP and $\mathrm{Ir} / \beta$-1,3-glucan-NP had fine biocompatibility for biological applications and they can effectively induce apoptosis and consequently result in the cell death through photocytotoxicity test.

3.5. Fluorescence Imaging In Vivo. The in vivo fluorescence imaging was studied to observe the distribution of the Ir/PGlc-NP and Ir/ $\beta$-1,3-glucan-NP in live rat, respectively. In this study, Ir/PGlc-NP or Ir/ $\beta$-1,3-glucan-NP was injected into mice via the tail vein and then we took observation of the mice after $24 \mathrm{~h}$. At $24 \mathrm{~h}$ after administration, the mice were killed to isolate the organs including liver, lung, heart, kidney, and spleen for fluorescence imaging and the images were shown in Figure 6. The pictures were obtained using optical and X-ray small animal imaging system and the colors with the order of red, orange, yellow, green, and blue in them represented the successive decrease in fluorescence intensity. Figures 6(a) and 6(b) indicated that the distribution of the Ir/PGlc-NP and $\mathrm{Ir} / \beta$-1,3-glucan-NP in organs was similar and the fluorescence intensity in the organs was clearly visualized. The fluorescence intensity distribution suggested that most of the nanoparticles have a tendency to be enriched in the liver; meanwhile, other organs like lung and kidney still have obvious fluorescence intensity distribution. The in vivo studies indicated that Ir (III) complexes packaged with two different water-soluble substances (PGlc, $\beta$-1,3-glucan) had no difference in the distribution in organs and that these two nanoparticles can serve as fluorescent drug tracer and fluorescent probe for different organs.

\section{Conclusions}

In conclusion, we reported the formation of two different water-soluble core-shell nanoparticles based on selfassembly. Morphological characterization of the nanoparticles was obtained by DLS and TEM which demonstrated that the nanoparticles were spherical in shape $(\sim 50 \mathrm{~nm}$ in diameter). The photophysics properties of Ir/PGlc-NP and $\mathrm{Ir} / \beta$-1,3-glucan-NP were systematically analyzed by PL emission spectra. In aqueous solutions, Ir/PGlc-NP and Ir/ $\beta-1,3-$ glucan-NP showed almost the same absorption and emission characteristics because of the similar molecular structure and nature properties. Upon coincubation of Ir/PGlc-NP and Ir/ $\beta$-1,3-glucan-NP with HepG2 cells, strong green fluorescence signals were mainly observed in cytoplasm of the cells. In photocytotoxicity experiment, with MTT assay nanoparticles caused effective apoptosis and death of HepG2 and Hela cells after irradiation at $445 \mathrm{~nm}$ for $30 \mathrm{~min}$. Furthermore, we observed the real-time fluorescence imaging of the live mice in vivo after injecting nanoparticles into them and the distribution of the Ir/PGlc-NP and Ir/ $\beta-1,3-$ glucan-NP in organs from the pictures at 24 after administration. Our results indicated the potential applications of the nanoparticles used as fluorescence probe for live tissue imaging.

\section{Conflict of Interests}

The authors declare that there is no conflict of interests regarding the publication of this paper. 


\section{Acknowledgments}

This work was financially supported by the National Basic Research Program of China (nos. 2012CB933301 and 2012CB723402), the Ministry of Education of China (no. IRT1148), NUPTSF (nos. NY215017, SZD2015014, and XYB2015245), and the National Natural Science Foundation of China (nos. 21222404, 61378081, 51173080, and 21104033).

\section{References}

[1] L. Zhou, J. L. Geng, G. Wang, J. Liu, and B. Liu, "A watersoluble conjugated polymer brush with multihydroxy dendritic side chains," Polymer Chemistry, vol. 4, no. 20, pp. 5243-5251, 2013.

[2] J. Liu, D. Ding, J. Geng, and B. Liu, "PEGylated conjugated polyelectrolytes containing 2,1,3-benzoxadiazole units for targeted cell imaging," Polymer Chemistry, vol. 3, no. 6, pp. 1567-1575, 2012.

[3] D. Ding, K. Li, Z. Zhu et al., "Conjugated polyelectrolytecisplatin complex nanoparticles for simultaneous in vivo imaging and drug tracking," Nanoscale, vol. 3, no. 5, pp. 1997-2002, 2011.

[4] C. F. Wu, T. Schneider, M. Zeigler et al., "Bioconjugation of ultrabright semiconducting polymer dots for specific cellular targeting," Journal of the American Chemical Society, vol. 132, no. 43, pp. 15410-15417, 2010.

[5] J. Schill, A. P. Schenning, and L. Brunsveld, "Self-assembled fluorescent nanoparticles from $\pi$-conjugated small molecules: en route to biological applications," Macromolecular Rapid Communications, vol. 36, no. 14, pp. 1306-1321, 2015.

[6] J. Liu, K. Li, J. L. Geng et al., "Single molecular hyperbranched nanoprobes for fluorescence and magnetic resonance dual modal imaging," Polymer Chemistry, vol. 4, no. 5, pp. 1517-1524, 2013.

[7] J. M. Behrendt, Y. Wang, H. Willcock et al., "Fluorescent nanoparticles from PEGylated polyfluorenes," Polymer Chemistry, vol. 4, no. 5, pp. 1333-1336, 2013.

[8] X. M. Lu, Q. L. Fan, G. W. Zhang, K. Y. Pu, and W. Huang, "Water-soluble light-emitting nanoparticles prepared by noncovalent bond self-assembly of a hydroxyl group functionalized oligo(p-phenyleneethynylene) with different water-soluble polymers," Science China Chemistry, vol. 53, no. 5, pp. 1122-1127, 2010.

[9] G. W. Zhang, X. M. Lu, Y. Y. Wang, Y. Q. Huang, Q. L. Fan, and W. Huang, "Water-soluble fluorescent nanoparticles without distinct aggregation of conjugated polymer chains," Polymer International, vol. 60, no. 1, pp. 45-50, 2011.

[10] T. Vokatá and J. H. Moon, "Synthesis of phenyleneethynylenedoped poly(p-phenylenebutadiynylene)s for live cell imaging," Macromolecules, vol. 46, no. 3, pp. 1253-1259, 2013.

[11] M. Twomey, Y. Na, Z. Roche et al., "Fabrication of coreshell nanoparticles via controlled aggregation of semiflexible conjugated polymer and hyaluronic acid," Macromolecules, vol. 46, no. 15, pp. 6374-6378, 2013.

[12] Y.-J. Ko, E. Mendez, and J. H. Moon, "Controlled aggregation in conjugated polymer nanoparticles via organic acid treatments," Macromolecules, vol. 44, no. 13, pp. 5527-5530, 2011.

[13] H. F. Shi, H. B. Sun, H. R. Yang et al., "Cationic polyfluorenes with phosphorescent iridium(III) complexes for time-resolved luminescent biosensing and fluorescence lifetime imaging,"
Advanced Functional Materials, vol. 23, no. 26, pp. 3268-3276, 2013.

[14] C. L. Yao, Z. Xue, M. Lian et al., "Phosphorescent iridium(III) complexes based on 2-phenylimidazo[1,2-a]pyridinetype ligands: synthesis, photophysical, electrochemical, and electrophosphorescent properties," Journal of Organometallic Chemistry, vol. 784, pp. 31-40, 2015.

[15] H. F. Shi, X. Ma, Q. Zhao et al., "Ultrasmall phosphorescent polymer dots for ratiometric oxygen sensing and photodynamic cancer therapy," Advanced Functional Materials, vol. 24, no. 30, pp. 4823-4830, 2014.

[16] P. F. Sun, X. M. Lu, Q. L. Fan et al., "Water-soluble iridium(III)containing conjugated polyelectrolytes with weakened energy transfer properties for multicolor protein sensing applications," Macromolecules, vol. 44, no. 22, pp. 8763-8770, 2011.

[17] H. B. Sun, J. Zhang, K. Y. Zhang et al., "Development of twochannel phosphorescent core-shell nanoprobe for ratiometric and time-resolved luminescence imaging of intracellular oxygen levels," Particle \& Particle Systems Characterization, vol. 32, no. 1, pp. 48-53, 2015.

[18] H. F. Shi, T. J. Tsuboi, S. J. Liu, Z. F. An, Q. Zhao, and W. Huang, "Multi-color poly(fluorenylene ethynylene)s with onchain phosphorescent iridium(III) complexes through energy transfer," Journal of Inorganic and Organometallic Polymers and Materials, vol. 25, no. 4, pp. 720-729, 2015.

[19] H. F. Shi, C. J. Xiujie, S. J. Liu et al., "Hyper-branched phosphorescent conjugated polyelectrolytes for time-resolved heparin sensing," ACS Applied Materials and Interfaces, vol. 5, no. 11, pp. 4562-4568, 2013.

[20] H. Takahashi, S.-I. Sawada, and K. Akiyoshi, "Amphiphilic polysaccharide nanoballs: a new building block for nanogel biomedical engineering and artificial chaperones," ACS Nano, vol. 5, no. 1, pp. 337-345, 2011.

[21] S. Li, Y. Y. Zhang, X. J. Xu, and L. N. Zhang, “Triple helical polysaccharide-induced good dispersion of silver nanoparticles in water," Biomacromolecules, vol. 12, no. 8, pp. 2864-2871, 2011.

[22] M. Ikeda, T. Hasegawa, M. Numata et al., "Instantaneous inclusion of a polynucleotide and hydrophobic guest molecules into a helical core of cationic $\beta$-1,3-glucan polysaccharide," Journal of the American Chemical Society, vol. 129, no. 13, pp. 3979-3988, 2007.

[23] M. Numata, C. Li, A.-H. Bae, K. Kaneko, K. Sakurai, and S. Shinkai, "Beta-1,3-glucan polysaccharide can act as a onedimensional host to create novel silica nanofiber structures," Chemical Communications, no. 37, pp. 4655-4657, 2005.

[24] M. C. Lin, P. F. Sun, G. S. Chen, and M. Jiang, "The glycostereoisomerism effect on hydrogelation of polymers interacting via dynamic covalent bonds," Chemical Communications, vol. 50, no. 68, pp. 9779-9782, 2014. 

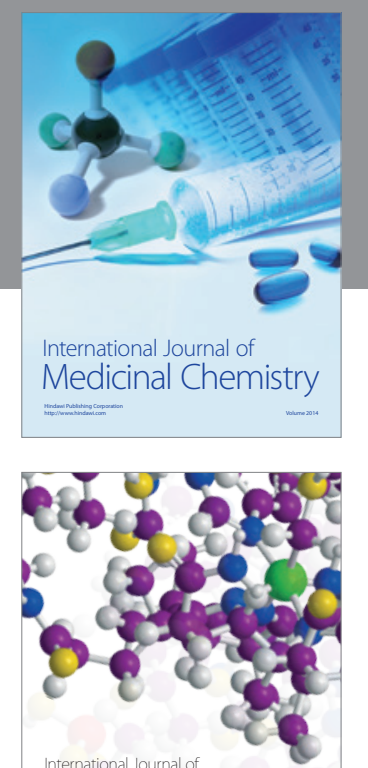

\section{Carbohydrate} Chemistry

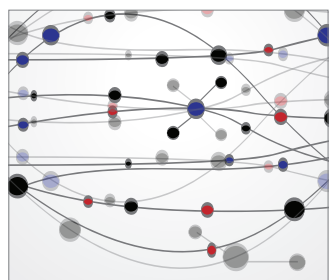

The Scientific World Journal
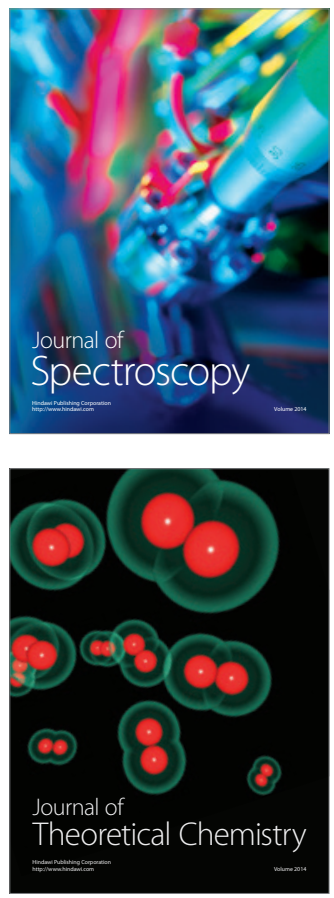
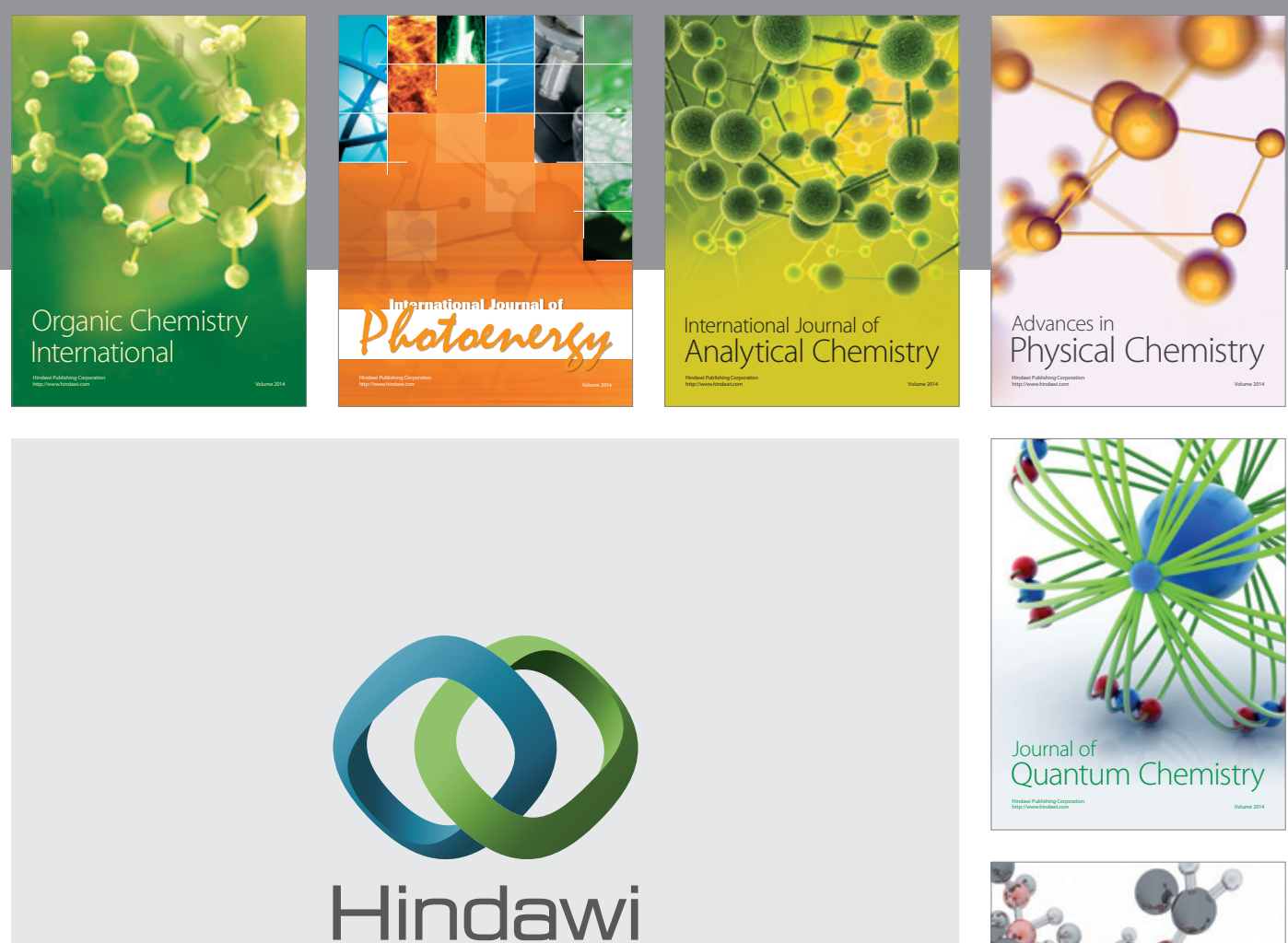

Submit your manuscripts at

http://www.hindawi.com

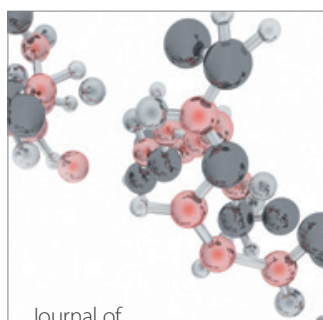

Analytical Methods

in Chemistry

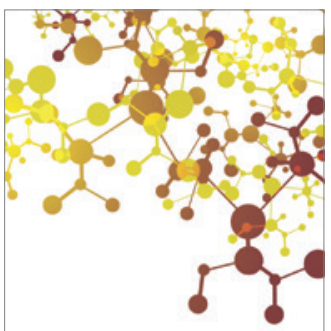

Journal of

Applied Chemistry

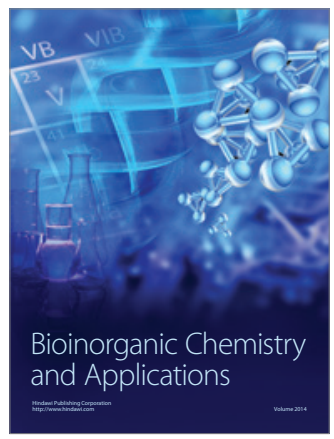

Inorganic Chemistry
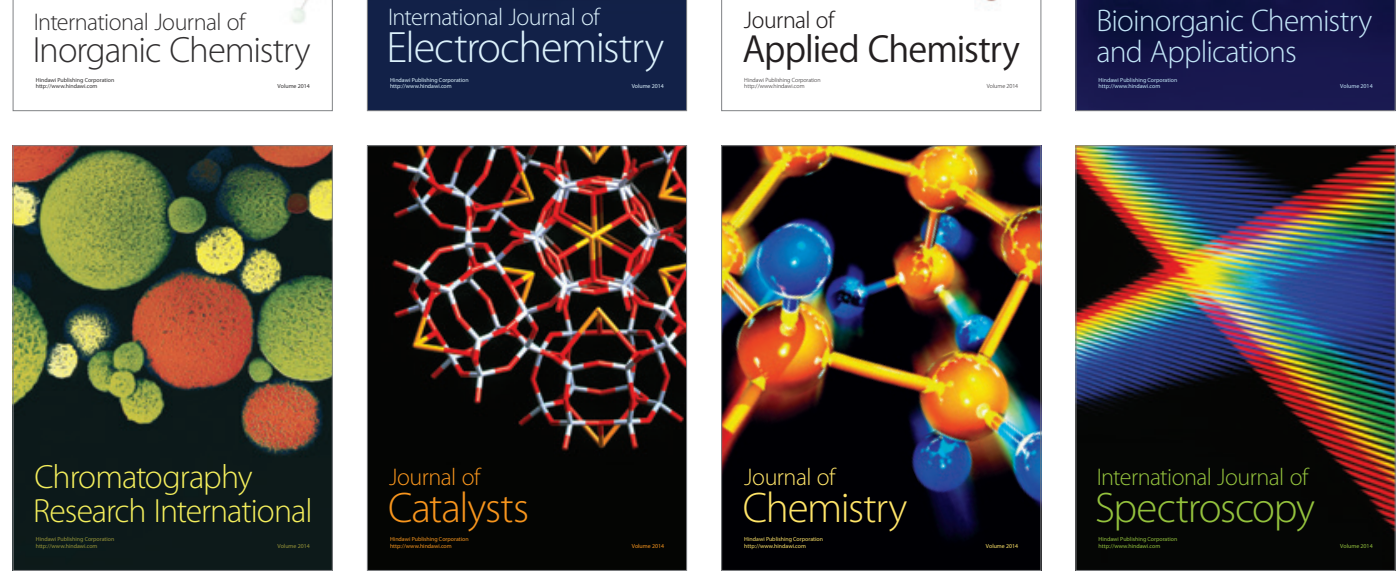